\title{
BMJ Open Group clinics for young adults with diabetes in an ethnically diverse, socioeconomically deprived setting (TOGETHER study): protocol for a realist review, co-design and mixed methods, participatory evaluation of a new care model
}

Chrysanthi Papoutsi, ${ }^{1}$ Dougal Hargreaves, ${ }^{2}$ Grainne Colligan, ${ }^{3}$ Ann Hagell, ${ }^{4}$ Anita Patel, ${ }^{3}$ Desirée Campbell-Richards, ${ }^{5}$ Russell M Viner, ${ }^{2}$ Shanti Vijayaraghavan, ${ }^{5}$ Martin Marshall, ${ }^{6}$ Trisha Greenhalgh, ${ }^{1}$ Sarah Finer ${ }^{3,5}$

To cite: Papoutsi C, Hargreaves D, Colligan G, et al. Group clinics for young adults with diabetes in an ethnically diverse, socioeconomically deprived setting (TOGETHER study): protocol for a realist review, co-design and mixed methods, participatory evaluation of a new care model. BMJ Open 2017;7:e017363. doi:10.1136/ bmjopen-2017-017363

\section{- Prepublication history} and additional material are available. To view these files please visit the journal online (http://dx.doi.org/ 10.1136/ bmjopen-2017-017363)

Received 19 April 2017 Accepted 24 April 2017

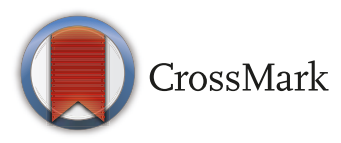

For numbered affiliations see end of article.

Correspondence to

Dr. Sarah Finer; s.finer@qmul. ac.uk

\begin{abstract}
Introduction Young adults with diabetes often report dissatisfaction with care and have poor diabetes-related health outcomes. As diabetes prevalence continues to rise, group-based care could provide a sustainable alternative to traditional one-to-one consultations, by engaging young people through life stage-, context- and culturally-sensitive approaches. In this study, we will co-design and evaluate a group-based care model for young adults with diabetes and complex health and social needs in socioeconomically deprived areas.
\end{abstract}

Methods and analysis This participatory study will include three phases. In phase 1, we will carry out a realist review to synthesise the literature on group-based care for young adults with diabetes. This theory-driven understanding will provide the basis for phase 2, where we will draw on experience-based co-design methodologies to develop a new, group-based care model for young adults (aged $<25$ years, under the care of adult diabetes services). In phase 3, we will use a researcher-in-residence approach to implement and evaluate the co-designed group clinic model and compare with traditional care. We will employ qualitative (observations in clinics, patient and staff interviews and document analysis) and quantitative methods (eg, biological markers, patient enablement instrument and diabetes distress scale), including a cost analysis.

Ethics and dissemination National Health Service ethics approval has been granted (reference 17/NI/0019). The project will directly inform service redesign to better meet the needs of young adults with diabetes in socioeconomically deprived areas and may guide a possible cluster-randomised trial, powered to clinical and cost-effectiveness outcomes. Findings from this study may be transferable to other long-term conditions and/or age groups. Project outputs will include briefing statements, summaries and academic papers, tailored for different audiences, including people living with diabetes, clinicians, policy makers and strategic decision makers.
Strengths and limitations of this study

- This study will draw on the strengths of group-based care to address the complex needs of young adults (aged 16-25 years) living with diabetes in ethnically diverse, socioeconomically deprived backgrounds.

- Cultural, developmental and practical considerations will be taken in account as part of iterative co-design and participatory evaluation of the new care model.

- A theory-driven, realist review of the literature will further inform the co-design process and will strengthen the transferability of findings.

- The project does not intend to generate an effect size, but findings may inform the design of a future cluster-randomised controlled trial.

Registration details PROSPERO (CRD42017058726).

\section{INTRODUCTION}

Diabetes has been described as one of the most significant global public health challenges of our time. ${ }^{1}$ Over the last four decades, the global prevalence of both type 1 and type 2 diabetes has increased sharply to reach an estimated $8.5 \%$ in the adult population in $2014 .^{2}$ In the UK, recent figures place the number of adults living with diabetes at 4.5 million, of whom around 1 million remained undiagnosed in 2016. ${ }^{3}$ This has raised the cost of diabetes care to $10 \%$ of the annual National Health Service (NHS) budget and has highlighted an urgent need to investigate different ways of delivering diabetes prevention and care. ${ }^{45}$ 
Despite growing emphasis on improving diabetes prevention and care in the adult population, less attention has been paid to young people living with diabetes. In England and Wales, over 27000 children and young people with diabetes receive care in paediatric diabetes units, ${ }^{6}$ and increasing numbers (poorly recorded) of adolescents and young adults are living with the condition. Young people constitute an important group for early intervention as good self-management practices internalised at a young age could persist throughout adulthood and reduce the risk of lifetime complications, prevent early mortality and lower costs for the health service. ${ }^{78}$ Young people of today will grow up to constitute the estimated 642 million living with diabetes worldwide in $2040 .{ }^{1}$ Yet, in $2014 / 2015$, only one quarter of children and young people with diabetes in England and Wales achieved recommended blood glucose control (HbAlc $<58 \mathrm{mmol} / \mathrm{mol}$ as per the National Institute for Health and Care Excellence 2004 target) and a similarly low proportion received all recommended care processes, with high variability between care providers. ${ }^{6}$ This is reinforced by research showing that mortality among young adults with diabetes in the UK is worse than in other European countries and rose significantly between 1990 and 2010. ${ }^{9}$ Diabetes is also known to have serious consequences in those diagnosed in childhood: diabetes-related complications (such as kidney and eye diseases) were seen in 1 in 3 of those with type 1 diabetes, and 3 in 4 with type 1 diabetes in their early 20s, within 8 years of diagnosis. ${ }^{10}$

Barriers to accessing healthcare for younger people have been well described, including lack of developmentally appropriate consultations, fear of being judged and stigmatised, lack of equitable access to services and diabetes-related distress. ${ }^{11}{ }^{12}$ Published data show that young adults report the worst NHS experience of any age group and have distinct healthcare needs and priorities compared with other age groups. ${ }^{13} 14$ This may be even more important for young people from socioeconomically deprived areas who achieve worse blood glucose control and present with more complications compared with more affluent areas and for those in ethnic minority groups who are disproportionately affected by type 2 diabetes. ${ }^{6}{ }^{15}$

The TOGETHER project will employ participatory methods to co-design, deliver and evaluate a model for diabetes care that addresses the needs of young people (aged 16-25 years) from socioeconomically deprived backgrounds. This model will draw on the strengths of group-based care, involving consultations and education sessions delivered by a multidisciplinary team and attended concurrently by a group of patients, in contrast to traditional one-to-one, consultant-led care.

Previous research has shown that group-based care in adults with diabetes resulted in better glycaemic control, problem-solving ability and quality of life and reduced time commitment for clinicians, compared with standard one-to-one consultations. ${ }^{16}{ }^{17}$ Systematic reviews on group care for diabetes showed additional benefits for clinical and patient-reported outcomes. ${ }^{18}{ }^{19}$ Further trials applying group-based care to diabetes and other conditions are underway with some work focusing on children and adolescents. ${ }^{20-22}$ However, these studies are limited to health but not social care needs, they have not utilised co-design processes or extensive user engagement and they are primarily targeting patient groups other than adolescents and young adults (aged $16-25$ years) in ethnically diverse, socioeconomically deprived backgrounds.

Our research seeks to address these uncertainties, including whether group-based care could be successfully adapted for this younger patient group and whether rigorous co-design of a new model of care can enhance acceptability and engagement. Furthermore, we will explore whether the positive impact of group-based education such as DAFNE (Dose-Adjustment for Normal Eating - a structured education programme for people withtype 1 diabetes) and DESMOND (Diabetes Education and Self Management for Ongoing and Newly Diagnosed - a structured education programme for people with type 2 diabetes) ${ }^{2324}$ as well as supporting evidence from studies of peer support ${ }^{2526}$ could be harnessed in group clinics for young adults to improve engagement with diabetes care and self-management in the wider context of individual, family, peer and social influences. ${ }^{27-29}$ This work will extend previous learning on the role of peer support groups and mentoring as a way to address complex health and social care issues. ${ }^{29-31}$

The TOGETHER project will be driven by the following aims and research questions:

\section{Aims}

1. To explore the scope, feasibility, impact and potential scalability of group clinics for young adults with diabetes and complex health and social care needs.

2. To contribute to NHS service re-design and improve care for people from hard-to-reach groups with longterm conditions.

\section{Research questions}

1. How and to what extent might an innovative, codesigned group clinic-based care model meet the complex health and social needs of young people with diabetes?

2. Could a group approach help support diabetes self-management? If so, what do the experiences of participants, the functioning of the group and the wider context in which the new model takes place tell us about its mechanisms of action?

3. What are the feasibility, acceptability, costs and impact on outcomes of introducing group clinics for their users and stakeholders? What is the organisational impact of this model to the NHS and other stakeholders?

4. What would be the optimal size and study design of a cluster-randomised controlled study to evaluate the clinical benefit and cost-effectiveness of offering 
group clinics to all suitable young adults with diabetes? What other factors should be considered when planning such an RCT (eg, factors relating to patient characteristics, existing models of service delivery, acceptability and mechanisms of action of group clinics on clinical outcomes)?

\section{METHODS AND ANALYSIS}

\section{Theoretical and conceptual framework}

Development and evaluation of new models of healthcare are frequently hindered by lack of robust, appropriate and explicit theoretical frameworks. ${ }^{32}$ The TOGETHER project will draw on a broad set of substantive social science and educational theories, including:

- ecological theories on supported self-management ${ }^{33-35}$

- work on patient expertise and experiential knowledge in practice p $^{3637}$

- critical education theory and experiential learning ${ }^{3839}$

- the concept of interdependency in figurational sociology ${ }^{40} 41$

The study will also draw on key theoretical and practical approaches to participatory research that allow researchers, practitioners and service users to learn together for the benefit of service re-design: ${ }^{42}{ }^{43}$

\section{(A) Participatory co-design}

The group clinics intervention will be iteratively co-designed using a participatory approach to ensure cultural, developmental and practical relevance; enhance recruitment and retention; and attempt to instigate system change and support sustainability. ${ }^{44}$

\section{(B) Participatory research and evaluation}

The 'researcher in residence' model will be adopted, as a practical manifestation of a participatory approach to research and evaluation. The model has three defining features: the researcher is an integral member of the frontline implementation team, their theoretical and practical contribution is explicit, along with their role to negotiate different bodies of expertise. ${ }^{45}$ The embedded researcher will bridge the qualitative and quantitative evaluations, helping to include practitioner and patient views into the design and feeding back early findings to stakeholders. The model has been applied successfully in a number of different settings. ${ }^{4546}$ A balance will be sought between how the 'researcher in residence' will be contributing to co-design and implementation in practice, and how this involvement will be translated in theoretical terms to increase transferable learning.

\section{Research plan}

The study will be conducted in three phases. A realist review will synthesise findings from existing literature to understand how group clinics may work for young adults with diabetes and other complex needs (phase 1). This understanding will be used to support a participatory co-design process with service users (phase 2). Following implementation of group-based care, the model will be evaluated using qualitative methods and quantitative methods (phase 3).

The timeline of the project and the processes followed are illustrated in figure 1 . The project is funded by the National Institute for Health Research Health Services and Delivery Research programme (ref. no. 15/25/20) to run for 3 years, from December 2016 to November 2019.

\section{Phase 1: realist review}

Following the Realist And Meta-narrative Evidence Syntheses: Evolving Standards (RAMESES), we will undertake a realist review to understand 'what works, for whom, under what circumstances' in group clinics for young adults with diabetes. ${ }^{47-49}$ The review will enable us to synthesise data from existing qualitative, quantitative and mixed-methods studies relevant to this topic area. Findings will support co-design, allow context-sensitive tailoring of the intervention and guide implementation and evaluation. ${ }^{50}$

Theoutputofthereviewwillbearealistprogrammetheory expressed in the form of Context-Mechanism-Outcome

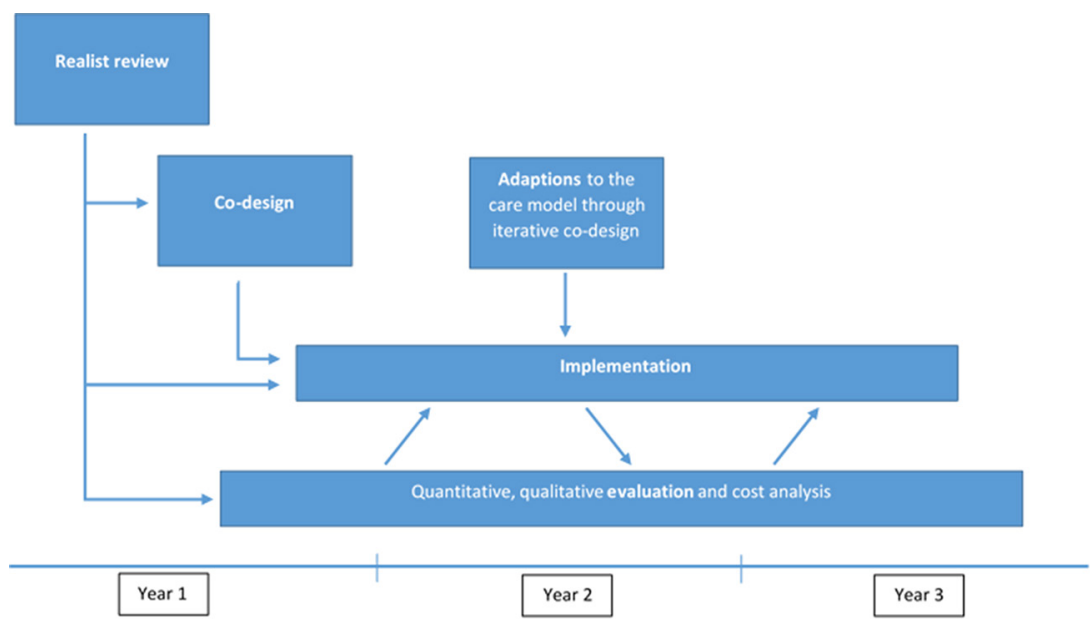

Figure 1 Overview of study design. 
Configurations (CMOCs) (see Glossary in supplementary files for definitions). We anticipate being able to examine specific mechanisms by which group clinics are deemed to 'work', including the potential for the group to generate a sense of greater security for individuals and to create norms, to harness social conformity and peer influence positively to change behaviour and to facilitate experiential learning and social support for self-care and engagement. To do this, we will build on recently completed realist reviews in the area ${ }^{51}{ }^{52}$ and will extend their findings for a young adult population with complex needs in socioeconomically deprived backgrounds.

The synthesis will follow five iterative stages: (A) locating existing theories, (B) searching for evidence, (C) selecting articles, (D) extracting and organising data and (E) synthesising the evidence and drawing conclusions. ${ }^{53}$

\section{(A) Locating existing theories}

An exploratory search of the literature along with articles provided by content experts will allow the development of an initial programme theory, that is, a set of provisional assumptions about how group clinics work for young adults with diabetes. This programme theory will continue to be refined throughout the review.

\section{(B) Searching for evidence}

The formal search strategy will be structured with the help of an information specialist and will be tested in MEDLINE to reach appropriate sensitivity and specificity. Free-text and indexing search terms will include those used by previous systematic reviews. ${ }^{51}{ }^{52}$ We will search the following databases: Embase, MEDLINE, MEDLINE-inprocess, PsycINFO, Web of Science, CENTRAL, Cochrane Database of Systematic Reviews, HTA database and ASSIA. Relevant studies will also be identified by hand searching, forward and backward citation tracking of key sources, contribution of key experts and grey literature where relevant. ${ }^{54}$ Results will be exported to Endnote for de-deduplication using automated and manual checking.

To respond to emerging findings (eg, on the importance of peer influence), we will carry out a second search as the review progresses to ensure we have covered all areas critical to continued engagement of young people in group clinics. This will allow us to progressively focus the review and enhance the explanatory depth of the analysis. Refinement of the programme theory will be discussed in research, co-design and project advisory meetings. This will allow us to link the review to practical questions that are critical in real world settings and provide added value to service delivery.

\section{(C) Screening and selecting articles}

Studies will be included if they focus on the introduction and delivery of group-based medical visits, group education and similar care delivery models for young people with diabetes. All study designs will be included across healthcare and community settings. Studies will be excluded if they focus solely on populations with considerable differences to young adults, for example, older people or very young children, if they discuss self-management education without a component of group interaction or if they are written in languages other than English. Inclusion and exclusion criteria will continue to be refined as needed throughout the review, according to best practice for realist reviews. ${ }^{47}$ Articles remaining after full-text screening will be classified according to their potential to contribute to programme theory. At the point of inclusion based on relevance, the trustworthiness and rigour of each study will be assessed as appropriate for each study design. ${ }^{47}$ A $10 \%$ random subsample of screening decisions will be reviewed by a second reviewer for consistency.

\section{(D) Extracting and organising data}

The main reviewer will read all articles classified as highly relevant, will extract descriptive study characteristics and will carry out manual coding for immersion in the data. Full texts will be uploaded on to NVivo V.11 (qualitative data management software) to continue coding in broad conceptual categories and to subsequently apply a realist logic of analysis. This means we will work iteratively to identify sections of text related to Contexts $(\mathrm{C})$, Mechanisms (M), Outcomes $(\mathrm{O})$ and the configurations between them (CMOCs), until theoretical saturation has been reached. In doing this, we will seek to interpret and explain how young adults with diabetes reason about and respond (by way of 'hidden' mechanisms) to 'resources' becoming available through group clinics and to identify the specific contexts or circumstances where these mechanisms are more likely to be 'triggered'. Again, a 10\% random subsample of coded articles will be reviewed by a second reviewer for consistency and disagreements will be solved by discussion.

\section{(E) Synthesising the evidence and drawing conclusions}

Coded data on CMOCs will be exported into Word documents to develop the final programme theory and the narrative of the synthesis. By moving between data and programme theory, we will be able to refine our explanations of why certain patterns seem to be occurring in certain contexts and under specific circumstances, related to group-based care. This will involve drawing on substantive theory to enhance the plausibility and coherence of the arguments. The final programme theory will consist of evidence-informed propositions, drawing on the literature, substantive theory, professional and patient expertise. These propositions will form the basis for co-design and implementation of group-based care in phase 2.

\section{Phase 2: co-design and implementation}

\section{(A) Co-design}

Health services often have limited success in changing health-related behaviours unless they take into account the perspectives and priorities of their patients and the staff providing that service. ${ }^{55}$ This is particularly true of 
patient groups (eg, those defined by age, ethnicity or deprivation) who are poorly served and/or reached by standard care models. The experience-based co-design process ${ }^{56}$ will bring together patients and staff into every stage of the project, supported by experienced co-design facilitators, allowing direct collaboration with the team who will be implementing the care models and regular opportunities for review and iteration. In this way, the co-design process will facilitate the development, review and refinement of user-centred services and care pathways. Co-design will be preceded by a brief national scoping exercise via professional groups to investigate current use and perceptions of group clinics in diabetes care, along with a formative evaluation to map existing local services for young adults with diabetes at two NHS Trusts.

We will recruit 15-20 young adults living with diabetes, members of their support network (eg, parents) and other stakeholders (eg, youth workers and healthcare professionals) to help us develop the group clinic model by drawing on their personal experience in co-design workshops. Participants will be identified via the diabetes service at Barts Health NHS Trust (Newham University Hospital), GP practices and lay support groups. Written information will be given explaining the study and what participation would involve. Co-design workshops will take place in community-based facilities in the London Borough of Newham, a deprived, ethnically diverse population with a high prevalence of long-term conditions and reduced life expectancy compared with UK averages. ${ }^{57}$

\section{(B) Implementation}

Once the co-designed model of care is established, all patients aged 16-25 years attending the Barts Health NHS Trust (Newham University Hospital) diabetes service will be offered the opportunity to attend group clinics. The 16-25 year age range incorporates adolescents and young adults with both type 1 and type 2 diabetes who are under the care of the multidisciplinary adult diabetes service during and after transition from paediatric services. The adult diabetes service involves close integrated working with local primary care and will facilitate referral into the group clinic model from GP care where relevant. We aim to recruit 80 young adults to this part of the research, using additional sites if required, giving a sample size that will enable sufficient development and evaluation of the new group clinic model, will allow feasibility testing and will guide a future, scaled-up cluster-randomised trial.

Each group clinic is expected to comprise around 8-10 individuals (young adults living with diabetes) and will be run by the Newham diabetes team, with support from related health and social care organisations. The content of these group clinics will be determined by the co-design process and the needs of the group but is likely to include a range of support for diabetes self-management (as already provided in current one-to-one clinics) and extend to a wider set of issues, including other health and social care needs and parental input where required. The content and running of the group clinics is anticipated to evolve during the 2 years in which they will run, due to refinements and revisions guided by ongoing co-design and participant feedback. The anticipated design of the group clinic model will follow two phases: (1) addition: where the group clinics are offered in addition to routine clinical care, and (2) substitution: to be rolled out after the addition model of group clinics has been implemented and considered to be running smoothly and safely by their participants, the clinical and research team and the project advisory group.

\section{Phase 3: evaluation}

(A) Qualitative methods

Qualitative methods will allow the research team to develop a better understanding of the contexts and mechanisms by which group clinics do, or do not, work. They will provide rich data on the experiences of patients in group-based care and their attitudes to managing their diabetes and interactions with health services in the wider context of individual, family, employment and other social factors. We will adopt a process-oriented and formative approach to the evaluation, negotiating the analysis and interpretation of the emerging evidence as implementation unfolds in order to maximise the impact of the intervention. Comparison of group clinics with traditional services will allow the relative merits of the new service to be better understood, given the established evidence that improvement interventions are more successful when practitioners can see a relative advantage over current practice. $^{58}$

\section{Data collection}

Data will be generated through a number of methods:

- Individual and group interviews with service users, group facilitators and practitioners: semistructured interviews will be conducted with a sample of service users including those who drop out of the groups and those who receive standard care. Sample size will be determined by data saturation and is expected to comprise between 20 and 30 participants. Purposive sampling will ensure variation in salient characteristics including type of diabetes, type of clinic attended, ethnicity and language. Sampling will also be driven by the findings of the realist review, as to the characteristics and circumstances that play a role in the success or failure of the group clinic model. Group interviews will be conducted with all group clinic facilitators and practitioners delivering standard care. With participant consent, interviews will be audiorecorded and transcribed. Bilingual health advocates will be used where necessary.

- Observations of group clinics and standard care: a sample of group clinics will be observed using a flexible pro forma to capture clinic characteristics such as session content, context, group dynamics and facilitation style. A sample of standard care consultations will be observed as a point of 
comparison. Detailed field notes will be kept during observations and audio recordings may be taken with participant consent. Group clinics and standard care appointments will be sampled to capture maximum variation (eg, morning/evening clinics in different areas).

- Documents: all documentation produced during codesign sessions, steering group meetings and group clinics will be collected for analysis. Service users and group facilitators will be asked to document their experiences inside and outside of clinics using photos, videos, other visual representations (eg, diagrams and drawings) and/or reflective journals.

\section{Data analysis}

All data will be analysed thematically using an iterative process of inductive and deductive coding. An initial list of codes will be generated a priori based on the realist review, the co-design stage and our research questions. Codes will be added to this initial list inductively as necessary. Data will be managed using NVivo V.11.

In combination with thematic analysis, the embedded researcher will apply a realist logic of analysis to the data to refine the realist programme theory deriving from the review in phase 1 . The programme theory will provide a platform for combining qualitative and quantitative data, primarily in relation to how quantitative outcomes can be linked to qualitatively described processes of change. This will allow us to address the question of how and why group clinics may work differently compared with standard care.

Emerging findings will be discussed with the wider research and co-design group so that the analysis will be co-created with practitioners and service users. Rigour will be enhanced by checking for negative cases that challenge emerging interpretations of the data.

\section{(B) Quantitative methods}

The detailed qualitative evaluation of the new care model will be complemented by a quantitative evaluation, which will investigate the potential impact of group clinics on clinical outcomes, processes and costs. Definitive evaluation of the impact of group clinics is not possible within this study as the intervention is not yet sufficiently developed, nor will it be delivered at a scale powered to investigate differences in clinical outcomes. The quantitative analysis will therefore (1) provide an early indication of the potential effects of group clinics on engagement and acceptability via attendance rates, measures of patient enablement and through use of the Problem Areas in Diabetes (PAID) scale, ${ }^{59-61}$ (2) investigate change in biological markers of diabetes control $\left(\mathrm{eg}, \mathrm{HbA}_{1 \mathrm{c}}\right.$ ) to guide sample size calculations for a future trial, (3) test feasibility of collecting service-level, resource (eg, staff contact and non-contact time), activity and process data for future unit-level comparisons in a cluster-randomised trial and (4) estimate costs associated with groups clinics compared with standard care.
The cost analysis will collate data on resource use (including routine and unscheduled care use) and will apply national unit costs to estimate staffing, capital and running costs related to running the standard versus group clinics at service level. Further analysis will look at the potential impact on use of other services and associated costs, and thus the extent to which the group clinic model substitutes for, rather than adds to, standard care. This in turn will provide an early indication of any potential for efficiency savings for the NHS. To best inform a future trial, all resource use and cost data will be presented in both aggregated and disaggregated forms and for different scenarios, for example, group clinic costs according to variations in attendance rates.

\section{Data analysis}

There will be different types of quantitative analysis of clinical outcomes and patient enablement:

- Intention to treat: simple descriptive statistics will be used to compare clinical outcomes among young adults with diabetes who were invited to attend the group clinics with controls from the same clinic over the previous year.

- Comparison of baseline characteristics, clinical outcomes and measures of patient enablement among patients who receive the intervention $(n=80)$ with those at additional external young adult diabetes clinics $(n=60)$ where the group clinics will not be implemented. The first stage of this process will complement qualitative findings on which patients are most attracted by the idea of group clinics. We will compare baseline sociodemographic and clinical characteristics of those who accept versus those who decline the opportunity to participate in group clinics. We will then use longitudinal data to compare the trajectories of clinical and enablement measures over the following year for individuals in the accept versus decline groups (difference in difference analysis).

- Unit-level data of clinical outcome (and indirect clinical outcome) data collected from additional external young adult diabetes clinics and National Diabetes Audit data (published annually by NHS Digital). This data analysis will inform a future scaledup cluster-randomised controlled trial of group clinics by (1) testing the feasibility of unit-level data collection, (2) identifying differences in the case mix of patients attending young adult diabetes clinics, and (3) characterising the clinical and process outcomes of young adults with diabetes under the care of different units.

\section{Ethics, safety and dissemination}

Ethics and safety

The study has been approved by the Office for Research Ethics Committees Northern Ireland (reference 17/ NI/0019). Standard rules apply for data security, confidentiality and information governance. Informed consent will be sought for ethnographic observations during group 
clinics, interviews and for accessing routinely collected NHS data on participants. Confidentiality and privacy between group clinic participants will be a priority, and all participants in the group clinics will be asked to sign a code of conduct to ensure that personal information is not shared outside of the group.

\section{Dissemination plan and project outputs}

Dissemination will be an ongoing process throughout the project, including activity at the outset to raise awareness of the project, at the mid-point to sustain interest and to feed back to participants and at the end and beyond to share learning. Our dissemination plan will build on our participatory approach to identify relevant audiences and maximise impact. We expect to target the following groups:

- project participants, user groups and local staff

- wider stakeholder community (including clinical networks)

- policy makers, strategic decision makers and funders

- academic community

The products needed to target these audiences will vary. We will write regular reports summarising our research activity and outputs, and these will be available via publicly accessible portals. We will prepare user-friendly versions of the main findings, briefing statements and policy summaries. Academic outputs will include journal articles in peer-reviewed, open access journals and conference presentations.

\section{DISCUSSION}

The challenge of improving outcomes for young adults with diabetes is particularly evident in ethnically diverse areas with high levels of socioeconomic deprivation and with an increasing burden of type 1 and type 2 diabetes. Traditional models of diabetes care, based on one-to-one clinic appointments with health professionals, do not meet the needs of these populations consistently, who may see their medical care as only one issue in a complex pattern of health and social care priorities. Although the ability to self-manage long-term conditions is increasingly considered to be the optimal means to achieve good health outcomes, this can lead to power struggles between patient and provider. ${ }^{62}$ There is a need to co-design and evaluate new care models that address diabetes care in the context of these wider needs and increasing demands, to offer alternative support in attaining self-management goals and engagement and to improve the experience and clinical outcomes of people living with diabetes.

As previous work has shown, group-based care requires 'reallocation of tasks, roles, and resources and a change in providers' attitudes from the traditional prescriptive approach to a more empathic role of facilitator ${ }^{93}$ This research will explore the different mechanisms that may or may not allow group clinic participants to engage with care that better suits their needs and improves their health outcomes. We will draw on previous literature to consider a range of potentially relevant factors: prior engagement with health services; appointment duration, frequency and flexibility; congruent social, peer and cultural contexts; and closer integration with other health and social care providers.

This study spans the innovation stage (co-design of a new service model) and early testing stage (evaluation of the acceptability, feasibility, costs and mechanisms of action of group clinic models) ${ }^{64}$ In contrast to studies that start with a clear idea of the intervention and evaluate the impact of introducing this intervention, we do not yet know the optimal role of, or the best way to implement, group clinics, especially among these patient groups in this geographical context. The care model for young adults with diabetes we propose to develop will evolve during the course of our study to ensure it is context sensitive and fit for purpose. For these reasons, our evaluation framework will be largely developmental combining qualitative and quantitative methods to best address our research questions. Project outcomes are expected to inform the potential design of a future cluster-randomised controlled trial to evaluate the impact of group clinics on clinical outcomes and cost-effectiveness.

\section{Author affiliations}

${ }^{1}$ Nuffield Department of Primary Care Health Sciences, University of Oxford, Oxford, UK

${ }^{2}$ UCL Great Ormond St. Institute of Child Health, University College London, London, UK

${ }^{3}$ Centre for Primary Care and Public Health, Blizard Institute, Barts and The London School of Medicine and Dentistry, Queen Mary University of London, London, UK

${ }^{4}$ Association for Young People's Health, London, UK

${ }^{5}$ Barts Health NHS Trust, London, UK

${ }^{6}$ Department of Primary Care and Population Health, University College London, London, UK

Correction notice This paper has been amended since it was published Online First. Owing to a scripting error, some of the publisher names in the references were replaced with 'BMJ Publishing Group'. This only affected the full text version, not the PDF. We have since corrected these errors and the correct publishers have been inserted into the references.

Contributors SF and DH conceptualised the study with input from MM, TG, AH, AP, DC-R, RMV, SV and GC. CP and SF wrote the first draft of this manuscript with significant input from DH, TG, MM, AH, AP, DC-R, RMV, SV and GC. All authors have read and approved the final manuscript.

Funding This work is supported by the National Institute for Health Research (NIHR) Health Services and Delivery Research (HS\&DR) programme (ref. no. 15/25/20).

Disclaimer The views and opinions expressed therein are those of the authors and do not necessarily reflect those of the National Institute for Health Research Health Services and Deliver Research programme, National Institute for Health Research, National Health Service or the Department of Health.

Competing interests None declared.

Patient consent The submitted paper is a study protocol.

Ethics approval Office for Research Ethics Committees Northern Ireland (reference 17/NI/0019).

Provenance and peer review Not commissioned; peer reviewed for ethical and funding approval prior to submission.

Data sharing statement Given this is a study protocol, no data are available to be shared yet. The authors will make appropriate provisions for data sharing and will include a statement in the paper describing the results of this study instead. 
Open Access This is an Open Access article distributed in accordance with the terms of the Creative Commons Attribution (CC BY 4.0) license, which permits others to distribute, remix, adapt and build upon this work, for commercial use, provided the original work is properly cited. See: http://creativecommons.org/ licenses/by/4.0/

(C) Article author(s) (or their employer(s) unless otherwise stated in the text of the article) 2017. All rights reserved. No commercial use is permitted unless otherwise expressly granted.

\section{REFERENCES}

1. International Diabetes Federation. Diabetes Atlas. 2015 http://www. diabetesatlas.org/ (Accessed 17 April 2017).

2. World Health Organisation. Global Report on Diabetes. $2016 \mathrm{http}: / /$ www.who.int/diabetes/global-report/en/ (Accessed 17 April 2017).

3. Diabetes UK. Prevalence Diabetes 2016. Available from: https:// www.diabetes.org.uk/Professionals/Position-statements-reports/ Statistics/Diabetes-prevalence-2016/ [Accessed 17 April 2017].

4. England NHS. NHS Diabetes Prevention Programme (NHS DPP. 2016 https://www.england.nhs.uk/ourwork/qual-clin-lead/diabetesprevention/ (Accessed 17 April 2017).

5. Hex N, Bartlett C, Wright D, et al. Estimating the current and future costs of type 1 and type 2 diabetes in the UK, including direct health costs and indirect societal and productivity costs. Diabet Med 2012;29:855-62.

6. Royal College of Paediatrics and Child Health. National Paediatric Diabetes Audit Report 2014-2015. Part 1: Care processes and outcomes. 2016 http://www.rcpch.ac.uk/improving-child-health/ quality-improvement-and-clinical-audit/national-paediatric-diabetesaudit-n-0\#2014-15\%20National\%20Paediatric\%20Diabetes\% 20Audit\%20reports (Accessed 17 April 2017).

7. Anderson BJ, Wolpert HA. A developmental perspective on the challenges of diabetes education and care during the young adult period. Patient Educ Couns 2004:53:347-52.

8. Northam EA, Todd S, Cameron FJ. Interventions to promote optimal health outcomes in children with type 1 diabetes-are they effective? Diabet Med 2006;23:113-21.

9. Viner RM, Hargreaves DS, Coffey C, et al. Deaths in young people aged 0-24 years in the UK compared with the EU15+ countries, 1970-2008: analysis of the WHO Mortality Database. Lancet 2014;384:880-92.

10. Dabelea D, Stafford JM, Mayer-Davis EJ, et al. Association of type 1 diabetes vs type 2 diabetes diagnosed during childhood and adolescence with complications during teenage years and young adulthood. JAMA 2017;317:825-35.

11. Kibbey KJ, Speight J, Wong JL, et al. Diabetes care provision: barriers, enablers and service needs of young adults with type 1 diabetes from a region of social disadvantage. Diabet Med 2013;30:878-84.

12. Tylee A, Haller DM, Graham T, et al. Youth-friendly primary-care services: how are we doing and what more needs to be done? The Lancet 2007;369:1565-73.

13. Hargreaves DS, Viner RM. Children's and young people's experience of the National Health Service in England: a review of national surveys 2001-2011. Arch Dis Child 2012;97:661-6.

14. Hargreaves DS, Sizmur S, Viner RM. Do young and older adults have different health care priorities? evidence from a national survey of English inpatients. J Adolesc Health 2012;51:528-32.

15. Khanolkar AR, Amin R, Taylor-Robinson D, et al. Ethnic minorities are at greater risk for childhood-onset type 2 diabetes and poorer glycemic control in England and Wales. J Adolesc Health 2016;59:354-61.

16. Trento M, Passera P, Borgo E, et al. A 5-year randomized controlled study of learning, problem solving ability, and quality of life modifications in people with type 2 diabetes managed by group care. Diabetes Care 2004;27:670-5.

17. Trento M, Passera P, Tomalino $M$, et al. Group Visits improve metabolic control in type 2 Diabetes. A 2-year follow-up 2001;24:995-1000.

18. Housden L, Wong ST, Dawes M. Effectiveness of group medical visits for improving diabetes care: a systematic review and metaanalysis. CMAJ 2013;185:E635-E644.

19. Edelman D, Gierisch JM, McDuffie JR, et al. Shared medical appointments for patients with diabetes mellitus: a systematic review. J Gen Intern Med 2015;30:99-106.

20. Rijswijk C, Zantinge E, Seesing F, et al. Shared and individual medical appointments for children and adolescents with type 1 diabetes; differences in topics discussed? Patient Educ Couns 2010;79:351-5
21. Mejino A, Noordman J, van Dulmen S. Shared medical appointments for children and adolescents with type 1 diabetes: perspectives and experiences of patients, parents, and health care providers. Adolesc Health Med Ther 2012;3:75-83.

22. Khan KM, Windt A, Davis JC, et al. Group Medical Visits (GMVs) in primary care: an RCT of group-based versus individual appointments to reduce $\mathrm{HbA1c}$ in older people. BMJ Open 2015;5:e007441.

23. DAFNE Study Group. Training in flexible, intensive insulin management to enable dietary freedom in people with type 1 diabetes: dose adjustment for normal eating (DAFNE) randomised controlled trial. BMJ 2002;325:746-9.

24. Davies MJ, Heller S, Skinner TC, et al. Effectiveness of the diabetes education and self management for ongoing and newly diagnosed (DESMOND) programme for people with newly diagnosed type 2 diabetes: cluster randomised controlled trial. BMJ 2008;336:491-5.

25. Blakemore SJ. The social brain in adolescence. Nat Rev Neurosci 2008:9:267-77.

26. Harden PN, Walsh G, Bandler N, et al. Bridging the gap: an integrated paediatric to adult clinical service for young adults with kidney failure. BMJ 2012;344:e3718.

27. Barry E, Roberts S, Oke J, et al. Efficacy and effectiveness of screen and treat policies in prevention of type 2 diabetes: systematic review and meta-analysis of screening tests and interventions. BMJ 2017;356:i6538.

28. Hargreaves DS, McVey D, Nairn A, et al. Relative importance of individual and social factors in improving adolescent health. Perspect Public Health 2013;133:122-31.

29. Greenhalgh T, Clinch M, Afsar N, et al. Socio-cultural influences on the behaviour of south asian women with diabetes in pregnancy: qualitative study using a multi-level theoretical approach. BMC Med 2015;13:120.

30. Greenhalgh T, Campbell-Richards D, Vijayaraghavan S, et al. New models of self-management education for minority ethnic groups: pilot randomized trial of a story-sharing intervention. J Health Serv Res Policy 2011;16:28-36.

31. Greenhalgh T, Collard A, Begum N. Sharing stories: complex intervention for diabetes education in minority ethnic groups who do not speak English. BMJ 2005;330:628.

32. Davidoff F, Dixon-Woods M, Leviton L, et al. Demystifying theory and its use in improvement. BMJ Qual Saf 2015;24:228-38.

33. Greenhalgh T. Chronic illness: beyond the expert patient. BMJ 2009;338:629-31.

34. Fisher EB, Brownson CA, O'Toole ML, et al. Ecological approaches to self-management: the case of diabetes. Am J Public Health 2005;95:1523-35.

35. Glass TA, McAtee MJ. Behavioral science at the crossroads in public health: extending horizons, envisioning the future. Soc Sci Med 2006;62:1650-71.

36. Pols J. Knowing patients. Science, Technology, \& Human Values 2013;39:73-97.

37. Mol A, Moser I, Pols J. Care in practice: on tinkering in clinics, homes and farms. Bielefeld: Transcript Verlag 2010.

38. Kolb DA. Experiential learning: experience as the source of learning and development. New Jersey: Pearson Education, 2014.

39. Freire P. Education for critical consciousness. London: Bloomsbury Publishing, 1973

40. Elias N. Problems of involvement and detachment. Br J Sociol 1956;7:226-52.

41. Elias N. The history of manners. Vol 1. Oxford: Basil Blackwell. 1978

42. Cornwall A, Jewkes R. What is participatory research? Soc Sci Med 1995:41:1667-76.

43. Marshall M, Mountford J. Developing a science of improvement. J R Soc Med 2013;106:45-50.

44. Jagosh J, Macaulay AC, Pluye P, et al. Uncovering the benefits of participatory research: implications of a realist review for health research and practice. Milbank Q 2012;90:311-46.

45. Marshall M, Pagel C, French $\mathrm{C}$, et al. Moving improvement research closer to practice: the Researcher-in-Residence model. BMJ Qual Saf 2014;23:801-5.

46. Eyre L, George B, Marshall M. Protocol for a process-oriented qualitative evaluation of the Waltham Forest and East London Collaborative (WELC) integrated care pioneer programme using the Researcher-in-Residence model. BMJ Open 2015;5:e009567.

47. Wong G, Greenhalgh T, Westhorp G, et al. Development of methodological guidance, publication standards and training materials for realist and meta-narrative reviews: the RAMESES (Realist and Meta-narrative evidence syntheses: evolving Standards) project. Health Serv Deliv Res 2014;2:1-252.

48. Wong G, Greenhalgh T, Westhorp G, et al. RAMESES publication standards: realist syntheses. BMC Medicine 2013;11:1-14. 
49. Pawson R. Evidence-based policy: a realist perspective. London: Sage, 2006.

50. Rycroft-Malone J, McCormack B, Hutchinson AM, et al. Realist synthesis: illustrating the method for implementation research. Implement Sci 2012;7:33.

51. Kirsh SR, Aron DC, Johnson KD, et al. A realist review of shared medical appointments: how, for whom, and under what circumstances do they work? BMC Health Serv Res 2017;17:113

52. Booth A, Cantrell A, Preston L, et al. What is the evidence for the effectiveness, appropriateness and feasibility of group clinics for patients with chronic conditions? A systematic review. Southampton UK: Health Services and Delivery Research, 2015.

53. Pawson R, Greenhalgh T, Harvey G, et al. Realist synthesis: an introduction. ESRC research methods programme Manchester: University of Manchester, 2004.

54. Greenhalgh T, Peacock R. Effectiveness and efficiency of search methods in systematic reviews of complex evidence: audit of primary sources. BMJ 2005;331:1064-5.

55. Piper D, ledema R, Gray J, et al. Utilizing experience-based codesign to improve the experience of patients accessing emergency departments in New South Wales public hospitals: an evaluation study. Health Serv Manage Res 2012;25:162-72.

56. The King's Fund. Experience-Based Co-design toolkit. 2013. https://www.kingsfund.org.uk/projects/ebcd (Accessed 17 April 2017).
57. London N. Newham's Local Economic Assessment 2010 to 2027. 2010. http://www.newham.info/lea (Accessed 17 April 2017).

58. Grol R, Dalhuijsen J, Thomas S, et al. Attributes of clinical guidelines that influence use of guidelines in general practice: observational study. BMJ 1998;317:858-61.

59. Howie JG, Heaney DJ, Maxwell M, et al. Quality at general practice consultations: cross sectional survey. BMJ 1999;319:738-43.

60. Howie JG, Heaney DJ, Maxwell M, et al. A comparison of a patient Enablement Instrument (PEI) against two established satisfaction scales as an outcome measure of primary care consultations. Fam Pract 1998;15:165-71.

61. Polonsky WH, Anderson BJ, Lohrer PA, et al. Assessment of diabetes-related distress. Diabetes Care 1995;18:754-60.

62. Snow R, Humphrey C, Sandall J. What happens when patients know more than their doctors? experiences of health interactions after diabetes patient education: a qualitative patient-led study. BMJ Open 2013;3:e003583.

63. Trento M, Gamba S, Gentile L, et al. Rethink Organization to iMprove education and outcomes (ROMEO): a multicenter randomized trial of lifestyle intervention by group care to manage type 2 diabetes. Diabetes Care 2010;33:745-7.

64. Craig P, Dieppe P, Macintyre S, et al. Developing and evaluating complex interventions: the New Medical Research Council guidance. BMJ 2008;337:a1655. 